\title{
Detecção e Reconhecimento de Minúcias em Imagens Datiloscópicas: Uma Abordagem Holística
}

\author{
Paulo S. de S. L. de Assumpção, Luziane F. de Mendonça \\ Programa de Pós-Graduação em Informática, Depto de Ciências de Computação - UFRJ, \\ CP 68530, CEP 21941-590, Rio de Janeiro, RJ \\ E-mail: paulosergio@cmb.gov.br, luziane@dcc.ufrj.br
}

\begin{abstract}
Resumo: A identificação por impressão digital é o método mais antigo e amplamente utilizado na garantia da autenticidade. Entretanto, a extração de forma confiável das minúcias é um desafio para muitos algoritmos, pois raramente as imagens capturadas possuem qualidade aceitável; contudo, boa parte das pesquisas se dedica apenas a uma das etapas do processamento. Este trabalho apresenta um sistema completo, desde a captura até o reconhecimento, utilizando estratégias de reconhecimento de padrões e técnicas de processamento de imagens para a extração dos pontos característicos. Algoritmos conhecidos na literatura são apresentados e avaliados utilizando um banco de imagens para verificar o desempenho da abordagem proposta.
\end{abstract}

Palavras-chave: impressão digital, processamento de imagem, biometria, extração de minúcias.

\section{Introdução}

Cada pessoa possui características próprias e exclusivas que a diferenciam das demais e compõem uma identidade individual específica. Do ponto de vista datiloscópico, esta peculiaridade é observada nas descontinuidades presentes nas papilas dérmicas, cujas linhas se interrompem ou se bifurcam abruptamente em uma estrutura de cristas e vales (tais formações são conhecidas como pontos de minúcias ou característicos). Com base nisso, a autenticidade pode ser expressa através do assinalamento de pelo menos 12 pontos de minúcias coincidentes e nenhum divergente após a comparação entre duas imagens de impressão digital.

Este trabalho apresenta um método, baseado nas ferramentas de processamento de imagem, para o reconhecimento automático de um datilograma através da extração dos pontos característicos. Um arranjo de algoritmos de processamento de imagem é apresentado com o objetivo de realçar as cristas e vales, localizar os pontos característicos, eliminar pontos espúrios, criar modelo de minúcias e reconhecer datilogramas.

Esse arranjo apresenta um processo constituído por um conjunto de métodos e filtros, conhecidos na literatura, sobrepostos e divididos em quatros etapas principais, as quais serão apresentadas nas subseções a seguir: após a captura, as imagens de impressão digital são préprocessadas visando à otimização da qualidade; os algoritmos de segmentação, normalização, filtragem e esqueletização são responsáveis por aprimorá-la. Em seguida, o processo de extração de pontos característicos localiza espacialmente as minúcias. Devido a ruídos remanescentes, uma etapa de pós-processamento visa eliminar as falsas características. Por fim, as imagens são reconhecidas através de seus vetores de minúcias.

\subsection{Segmentação}

Inicialmente, a segmentação [7] é empregada para separar as regiões de primeiro plano da imagem (área de interesse, composta pelas cristas e vales) das regiões do fundo (fora das fronteiras da área de impressão digital, sem qualquer informação válida), as quais serão descartadas. A decisão 
sobre o descarte é baseada no valor da variância $V(k)$ do bloco $k$ da imagem ${ }^{1}$, cujo valor varia significativamente de acordo com a qualidade da imagem de impressão digital a ser processada. Portanto, o valor referente ao limiar $V_{t o l}$ deve levar em conta as características da imagem.

Para tanto, o método da coerência [2] pode ser aplicado para indicar a intensidade dos gradientes em um bloco centrado no ponto $(i, j)$ ao longo da crista. Uma vez que a impressão digital é constituída principalmente de estruturas de linhas paralelas, a coerência será maior no primeiro plano. Assim, são descartados os pixels cujos valores de $V(k)$ são superiores a $l(k)$.

$$
l(k)=\frac{\sqrt{\left(G_{x x}-G_{y y}\right)^{2}+4 G_{x y}^{2}}}{G_{x x}+G_{y y}}
$$

onde $G_{x}$ e $G_{y}$ são as estimativas do gradiente local obtidos através do método de Sobel [8] e são usados para encontrar os valores de $G_{x x}=\sum_{k} G_{x}^{2}, G_{y y}=\sum_{k} G_{y}^{2}$ e $G_{x y}=\sum_{k} G_{x} G_{y}$.

\subsection{Normalização}

A normalização [6] padroniza o intervalo de valores de nível de cinza dos pixels de uma imagem de modo que permaneça em uma faixa de valores desejados. O nível de cinza normalizado no pixel $(i, j), N(i, j)$, depende dos valores calculados a partir da imagem $I$ segmentada e dos valores desejados, respectivamente: médias $M$ e $M_{0}$ e variâncias $V A R$ e $V A R_{0}$. Em (1), temos que $\beta=1$ se $I(i, j)>M$; caso contrário, $\beta=-1$.

$$
N(i, j)=M_{0}+\beta \sqrt{\frac{V A R_{0}(I(i, j)-M)^{2}}{V A R}}
$$

\subsection{Filtragem}

O desempenho dos algoritmos de extração de minúcias depende muito da qualidade das imagens. Com isso, filtros podem ser aplicados de modo a destacar as características em uma determinada orientação e frequência. As etapas para a obtenção da imagem filtrada estão descritas a seguir.

\subsubsection{Estimativa da Orientação}

A orientação [6], $O(i, j)$, representa a direção da crista no ponto $(i, j)$, obtida através do ângulo da tangente à posição da minúcia em relação ao eixo horizontal (direção das cristas). Sua obtenção é descrita a seguir, iniciando com a divisão da imagem em blocos $w \times$ w

1. Para cada bloco, utilizando o operador de Sobel [8], estime os gradientes $\partial_{x}(i, j)$ e $\partial_{y}(i, j)$, os quais representam as magnitudes nas direções $x$ e $y$, respectivamente.

2. A orientação do bloco centrado no pixel $(i, j)$ é dada por $\theta(i, j)=\frac{1}{2} \tan ^{-1} \frac{V_{y}(i, j)}{V_{x}(i, j)}$, em que

$$
V_{x}(i, j)=\sum_{u=i-\frac{w}{2}}^{i+\frac{W}{2}} \sum_{v=j-\frac{w}{2}}^{i+\frac{W}{2}} 2 \partial_{x}(u, v) \partial_{y}(u, v) \quad V_{y}(i, j)=\sum_{u=i-\frac{w}{2}}^{i+\frac{W}{2}} \sum_{v=j-\frac{w}{2}}^{i+\frac{W}{2}} \partial_{x}(u, v)^{2}-\partial_{y}(u, v)^{2} .
$$

3. A orientação da imagem é convertida num campo de vetores contínuos, em que $\Phi_{x}(i, j)=$ $\cos (2 \theta(i, j))$ e $\Phi_{y}(i, j)=\sin (2 \theta(i, j))$ são os componentes $x$ e $y$ do campo, respectivamente.

4. Então, o filtro Gaussiano é aplicado para suavizar o campo de orientação via expressão a seguir, em que $G$ é o núcleo formado pelo filtro Gaussiano de tamanho $w_{\Phi} \times w_{\Phi}$ :

$$
\Phi_{r}^{\prime}(i, j)=\sum_{u=-\frac{w_{\Phi}}{2}}^{\frac{w_{\Phi}}{2}} \sum_{v=-\frac{w_{\Phi}}{2}}^{\frac{w_{\Phi}}{2}} G(u, v) \Phi_{r}(i-u w, j-v w), \quad r=x, y .
$$

Por fim, a orientação suavizada no pixel $(i, j)$ é definida como $O(i, j)=\frac{1}{2} \tan ^{-1} \frac{\Phi_{x}^{\prime}(i, j)}{\Phi_{y}^{\prime}(i, j)}$.

\footnotetext{
${ }^{1}$ Valor médio do quadrado da diferença entre o nível de cinza nos pontos $(i, j)$ e a média do nível de cinza no bloco $k$.
} 


\subsubsection{Estimativa de Frequência}

Os níveis de cinza ao longo das cristas podem ser modelados como uma forma de onda senoidal ao longo de uma direção ortogonal à orientação. Portanto, a frequência [6] da crista é uma propriedade intrínseca de uma impressão digital e pode ser encontrada utilizando os passos a seguir, iniciando com a divisão da imagem de blocos de tamanho $w \times w$.

1. Para cada bloco centrado no pixel $(i, j)$, calcule uma janela orientada de tamanho $l \times w$ (o valor padrão é $l=32$ ), de acordo com

$$
u=i+\left(d-\frac{w}{2}\right) \cos \theta(i, j)+\left(k-\frac{l}{2}\right) \sin \theta(i, j) ; \quad v=j+\left(d-\frac{w}{2}\right) \sin \theta(i, j)+\left(\frac{l}{2}-k\right) \cos \theta(i, j) .
$$

Calcule também a assinatura $X[k]=\frac{1}{W} \sum_{d=0}^{w-1} G(u, v)$ para $k=0, \ldots, l-1$.

2. Se não houver minúcias na janela orientada, a assinatura $X$ constitui uma forma senoidal discreta. Logo, a frequência das cristas pode ser estimada a partir da assinatura $X$ : $\Omega(i, j)=\frac{1}{\tau(i, j)}$ onde $\tau(i, j)$ é o número médio de pixels entre dois picos consecutivos.

3. Os blocos não contemplados no item anterior (devido a pontos característicos ou cristas e vales corrompidos) terão seus valores de frequência determinados via interpolação das frequências dos blocos vizinhos (com forma senoidal bem definida). Logo $\Omega(i, j)=a / b$ em que $W_{g}$ é um núcleo Gaussiano discreto com média 0 e variância $9, w_{\Omega}=7$ é o tamanho do núcleo, $\mu(x)=\max \{0, x\}, \delta(x)=\max \{0, \operatorname{sgn}(x)\}$ e

$$
a=\sum_{u-\frac{w_{\Omega}}{2}}^{\frac{w_{\Omega}}{2}} \sum_{v-\frac{w_{\Omega}}{2}}^{\frac{w_{\Omega}}{2}} W_{g}(u, v) \mu(\Omega(i-u, j-v)) ; \quad b=\sum_{u-\frac{w_{\Omega}}{2}}^{\frac{w_{\Omega}}{2}} \sum_{v-\frac{w_{\Omega}}{2}}^{\frac{w_{\Omega}}{2}} W_{g}(u, v) \delta(\Omega(i-u, j-v)+1) .
$$

4. As distâncias entre as cristas mudam suavemente entre as vizinhanças; por isso, um filtro passa-baixa é utilizado para remover os valores atípicos (outliers) de acordo com (3), onde $W_{l}$ é o filtro Gaussiano com núcleo de tamanho $w_{l}=7$.

$$
F(i, j)=\sum_{u-\frac{w_{\Omega}}{2}}^{\frac{w_{l}}{2}} \sum_{v-\frac{w_{\Omega}}{2}}^{\frac{w_{l}}{2}} W_{l}(u, v) \Omega(i-u w, j-v w)
$$

\subsubsection{Filtro de Gabor}

Para melhorar a imagem de impressão digital é aplicado um filtro de passa-banda que deixará presente na imagem apenas os valores dos sinais correspondentes a uma frequência desejada. Com isso, é possível realçar certas características e eliminar ruídos ou imperfeições presentes em uma frequência conhecida. A equação (4) apresenta a aplicação do filtro de Gabor [3] sobre a imagem do datilograma,

$$
E(i, j)=\sum_{u=-\frac{w_{x}}{2}}^{\frac{w_{x}}{2}} \sum_{v=-\frac{w_{y}}{2}}^{\frac{w_{y}}{2}} G(u, v ; O(i, j) ; F(i, j)) N(i-u, j-v)
$$

em que $O(i, j)$ é o valor da orientação no ponto $(i, j), F(i, j)$ o valor da sua frequência, $x_{\theta}=$ $x \cos \theta+y \sin \theta$ e $y_{\theta}=-x \sin \theta+y \cos \theta$ orientam a aplicação do filtro de acordo com a direção da crista, e $G(x, y ; \theta, f)=\exp \left\{-\frac{1}{2}\left[\frac{x_{\theta}^{2}}{\sigma_{x}^{2}}+\frac{y_{\theta}^{2}}{\sigma_{y}^{2}}\right]\right\} \cos \left(2 \pi f x_{\theta}\right)$.

Os valores de $\sigma_{x}$ e $\sigma_{y}$ representam os desvios padrão da envoltória gaussiana que está sobre os eixos $x$ e $y$. Alterar esses valores implica diretamente na faixa de passagem do filtro (bandwidth). Com valores maiores, maior será a robustez do filtro perante ruídos, mas em contrapartida há uma redução na faixa de passagem. Diminuir $\sigma_{x}$ e $\sigma_{y}$ fará com que haja um aumento na faixa de passagem e corre-se o risco de ser seletivo demais na frequência, inserindo pontos espúrios na imagem. Com isso, $\sigma_{x}$ e $\sigma_{y}$ podem ser calculados como $\frac{1}{2} F(i, j)$, onde $F$ é a frequência. 


\subsection{Binarização}

A maioria dos algoritmos de extração de minúcias opera em imagens binárias, constituídas por pixels pretos (cristas) e brancos (vales). A binarização é um processo que converte uma imagem em escala de cinza em uma imagem binária, para melhorar o contraste entre as cristas e vales e auxiliar na extração de minúcias. As etapas da binarização estão listadas a seguir, iniciando com a divisão da imagem em blocos de tamanho $w \times w$.

1. Cálculo da média do bloco de acordo $M(k)=\frac{1}{w^{2}} \sum_{m=0}^{w} \sum_{n=0}^{w} E(i+m, j+n)$, em que $E$ é a imagem filtrada.

2. Se o valor do pixel $(i, j)$ for maior do que a média do bloco considerado, ele receberá o valor 0 (preto); caso contrário, receberá o valor 1 (branco).

\subsection{Esqueletização}

A etapa final de aprimoramento da imagem é o desbaste das cristas. A esqueletização é uma operação morfológica que corrói sucessivamente os pixels que compõem o primeiro plano até que as linhas fiquem com um pixel de espessura, preservando a conectividade das estruturas da crista enquanto forma uma versão esqueletizada da imagem binária.

O método de Hilditch [5] é utilizado para decidir se o valor do ponto $p=(i, j)$ deve ser mudado de preto (0) para branco (1). Inicialmente, devemos considerar a vizinhança em torno do ponto; para decidir se ele deve ser retirado ou mantido devemos caminhar pela vizinhança no sentido horário para obter o número $B(p)$ de vizinhos de $p$ diferentes de 0 e o número $A\left(p_{i}\right)$ de transições de 0 para 1 (sequência horária) de um vizinho $p_{i}$ de $p$.

O algoritmo deve mudar a cor do pixel se este satisfizer: $(I) 2 \leq B(p) \leq 6 ; \quad(I I) A\left(p_{1}\right)=$ $1 ; \quad(I I I) p_{2} p_{4} p_{8}=0$ ou $A\left(p_{1}\right) \neq 1 ; \quad(I V) p_{2} p_{4} p_{6}=0$ ou $A\left(p_{3}\right) \neq 1$.

Ou seja, de $(\mathrm{I})$, se $B(p)=1$ então $p$ é um ponto do esqueleto; $B(p)=0, p$ é um ponto isolado; se $B(p)=7$, então $p$ não é mais a fronteira do padrão; nesses três casos, $p$ deve ser mantido. A condição (II) é um teste de conectividade: quando $A\left(p_{1}\right)>1$ é possível perceber que alterando $p_{1}$ para 0 o padrão será desconectado. A condição (III) garante que não ocorram erosões em linhas verticais de 2 pixels de largura. Por fim, a última condição garante que linhas horizontais com 2 pixels de largura não sejam corroídas pelo algoritmo.

\subsection{Extração de Minúcias}

A abordagem baseada no número de cruzamentos (Crossing Number) [1] aplicada diretamente sobre a imagem esqueletizada é a mais comumente empregada no processo de extração de minúcias. Assim, o número de cruzamentos do pixel $p$ é definido como a metade da soma das diferenças entre pares de pixels adjacentes que definem as 8 casas vizinhas $p_{1}, \ldots, p_{8}$ de $p$, ou seja, $c n(p)=\frac{1}{2} \sum_{i=1}^{k} p_{1}-p_{i+1}$. Utilizando as propriedades do número de cruzamento, o pixel da crista pode ser classificado conforme a Tabela 1.

Tabela 1: Propriedades do número de cruzamentos (CN)

\begin{tabular}{|c|c|c|c|c|c|}
\hline CN & 0 & 1 & 2 & 3 & 4 \\
\hline Prop. & Pt. isolado & Terminação & Continuidade da crista & Bifurcação & Cruzamento \\
\hline
\end{tabular}

\subsubsection{Remoção de Pontos Espúrios}

Após a extração dos pontos característicos, é necessário eliminar as falsas minúcias oriundas dos ruídos presentes na imagem. O algoritmo [10] analisa uma vizinhança $w \times w$ para cada ponto marcado, e decide se ele representa uma falsa minúcia ou não. Para cada minúcia marcada como candidata à terminação ou bifurcação, inicialize com 0 uma máscara $M$ de tamanho 
$w \times w$. Cada pixel de $M$ corresponde ao pixel da imagem afinada centrada no ponto onde a minúcia foi marcada. Rotule com -1 o pixel central de $M$ (candidato à minúcia na imagem afinada). Para uma terminação, faça:

1. Rotule com 1 todos os pixels na máscara $M$ conectados com a terminação.

2. Faça uma volta completa sobre a borda da máscara $M$ (sentido horário) e verifique o estado dos pixels fazendo $T_{01}$ igual ao número de transições de 0 para 1 encontradas. Se $T_{01}=1$, a terminação é verdadeira.

Para uma bifurcação, faça:

1. Faça uma volta completa (sentido horário) pela vizinhança da bifurcação e rotule os três pixels conectados a este como 1,2 e 3. Para cada pixel rotulado com 1, deve-se procurar seus componentes conexos dentro de $M$, ou seja, os pontos pretos que permitam a conexão com esse pixel. A rotulação deve ser realizada com valores de 1 até o final de $M$ ou até encontrar outro valor de rótulo. O mesmo deve ser feito para os demais segmentos.

2. Conte o número de transições de 0 para 1, 2 e 3 encontradas quando se realiza uma volta completa seguindo a borda da máscara $M$ (sentido horário). O número de transições será marcado como $T_{01}, T_{02}$ e $T_{03}$. Se forem iguais a 1 , então a bifurcação é verdadeira.

\subsection{Reconhecimento}

O reconhecimento da impressão digital [4] consiste na verificação dos elementos comuns pertencentes aos dois mapas de minúcias. Cada minúcia possui informação relativa à sua posição espacial, tipo e direção. Uma vez realizado o alinhamento entre duas impressões digitais, se elas se tratarem de imagens referentes ao mesmo dedo, as minúcias detectadas em cada imagem estarão próximas. Para comparar esses dois mapas de minúcias, considere dois vetores, um pertencente a uma imagem modelo $T$ e outro pertencente a uma imagem $E$, escritos como $T=\left(m_{1}, m_{2}, \ldots, m_{n}\right)$ e $E=\left(m_{1}^{\prime}, m_{2}^{\prime}, \ldots, m_{n}^{\prime}\right)$ onde $m$ e $m^{\prime}$ são os elementos extraídos de cada minúcia. Para se comparar esses dois mapas pode ser tomada a distância euclidiana entre as minúcias dos vetores $T$ e $E$. Uma minúcia $m_{j}$ de $T$ será considerada a mesma que uma minúcia $m_{i}$ de $E$ se a distância euclidiana entre elas for menor do que uma tolerância $r_{0}$.

Além do critério da distância, deve-se ter a diferença entre a orientação das minúcias $d_{d}$ menor do que uma tolerância angular $\theta_{0}$, ou seja, $d_{d}\left(m_{j}^{\prime}, m_{i}\right)=\min \left(\left|\theta_{j}^{\prime}-\theta_{i}\right|,\left(360^{\circ}-\left|\theta_{j}^{\prime}-\theta_{i}\right|\right)\right) \leq \theta_{0}$.

Os mapas $T$ e $E$ deverão ser alinhados para maximizar o número de minúcias reconhecidas. O alinhamento será realizado mantendo $T$ fixo e corrigindo as posições das minúcias de $E$, de modo a sobrepor um mapa sobre o outro. A rotação entre os mapas será corrigida através da diferença entre as orientações das minúcias. Os passos para o reconhecimento entre minúcias são descritos a seguir:

1. Obtenha a distância relativa entre os pares de minúcias através da equação que representa o quanto a imagem modelo está transladada. Tome a diferença entre os ângulos que indicam a direção das minúcias $\Delta \theta=\theta^{\prime}-\theta$.

2. Faça o alinhamento entre as minúcias da imagem de entrada utilizando a equação (5).

$$
\left[\begin{array}{l}
x_{i}^{A} \\
y_{i}^{A} \\
\theta_{i}^{A}
\end{array}\right]=\left[\begin{array}{c}
\Delta x \\
\Delta y \\
\Delta \theta
\end{array}\right]+\left[\begin{array}{ccc}
\cos \Delta \theta & \sin \Delta \theta & 0 \\
\cos \Delta \theta & \sin \Delta \theta & 0 \\
0 & 0 & 1
\end{array}\right]\left[\begin{array}{c}
x_{i}-x^{d} \\
y_{i}-y^{d} \\
\theta_{i}-\theta^{d}
\end{array}\right]
$$

3. Uma vez alinhados os dois vetores planares, é tomada a distância euclidiana entre as minúcias próximas que possuam a diferença entre as direções menores que a tolerância.

Após realizar a comparação do mapa de minúcias entre duas impressões digitais, o número de reconhecimento de minúcias é obtido dividindo o número de minúcias reconhecidas pela quantidade de minúcias encontradas. 


\section{Resultados}

Para verificar a precisão do arranjo de algoritmos apresentado foi utilizado um banco de dados privado com 10000 imagens impostoras e 30 genuínas (Figura 1). As Eq. 6 apresentam os cálculos da Taxa de Falsa Aceitação e Falsa Rejeição baseadas no número de amostras do banco de dados e na quantidade de comparações realizadas (impostoras e genuínas). Assim, procura-se o ponto de equilíbrio, representado pela interseção das curvas de TFA e TFR, a que chamamos Taxa de Intersecção de Erros (TIE ou Error Equal Rate - EER). Quanto mais baixo for o TIE mais preciso é um sistema biométrico

$$
T F A(t)=\frac{\operatorname{card}\{g m s \mid g m s<t\}}{\operatorname{card}\{g m s\}} \quad \operatorname{TFR}(t)=\frac{\operatorname{card}\{i m s \mid i m s \geq t\}}{\operatorname{card}\{i m s\}}
$$

onde ims (Impostor Matching Score) e gms (Genuine Matching Score) são os vetores que contem os valores obtidos com a comparação entre as imagens de dedos diferentes e iguais, respectivamente; $t$ é o valor da pontuação; e $\operatorname{card}\{x\}$ é a cardinalidade do vetor $x$.

A Tabela 2 apresenta os resultados obtidos por alguns sistemas de reconhecimento de impressão digital que participaram da competição internacional Fingerprint Verification Competition, em 2006 [9].

Tabela 2: Valores de TIE

\begin{tabular}{|c|c|c|c|c|c|c|c|c|c|c|}
\hline ID & P088 & P015 & P009 & P058 & P101 & P066 & P065 & P045 & P067 & P141 \\
\hline TIE(\%) & 0,021 & 0,032 & 0,095 & 0,100 & 0,121 & 0,122 & 0,137 & 0,138 & 0,185 & 0,237 \\
\hline
\end{tabular}

Os valores para TFA, TFR e TIE obtidos no uso do algoritmo apresentado são de 0,8\%, de $18,6 \%$ e de $0,066 \%$, respectivamente (Figura 2). Comparativamente, é possível observar que o algoritmo proposto promove a melhora da qualidade da imagem da impressão digital, realçando os vales e as cristas; além disso, tal metodologia mostra-se competitiva se comparada com os valores da Tabela 2, os quais indicam a precisão em uma competição de algoritmos de identificação biométrica.

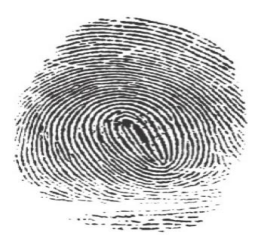

(a)

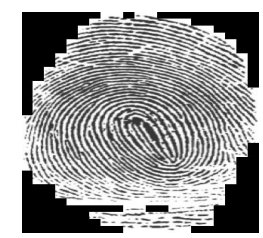

(b)

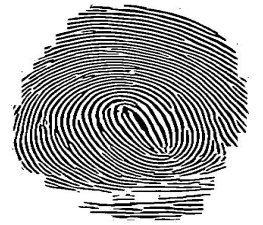

(f)

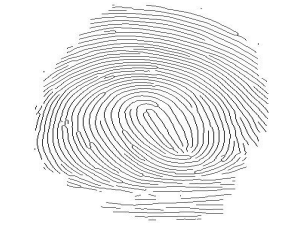

$(\mathrm{g})$

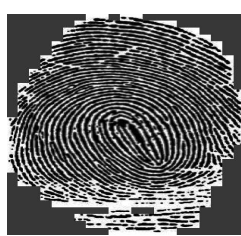

(c)

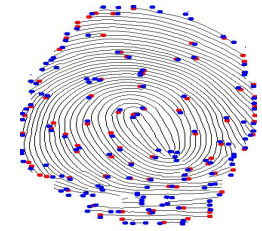

(h)

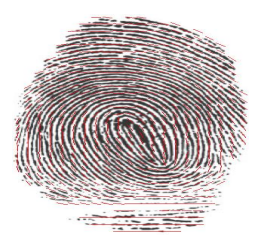

(d)

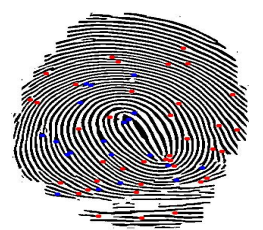

(i)

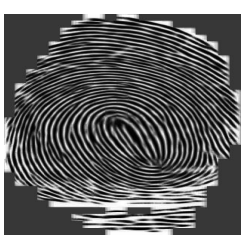

(e)

Figura 1: Resultado do processamento. (a) original, (b) segmentação, (c) normalização, (d) orientação, (e) filtragem, (f) binarização, (g) esqueletização, (h) extração de minúcias e (i) remoção de pontos espúrios

\section{Conclusão}

Neste trabalho, um conjunto de algoritmos baseados em modelos matemáticos para o reconhecimento de padrões, análise estatística e processamento de imagens, são adaptados da literatura 


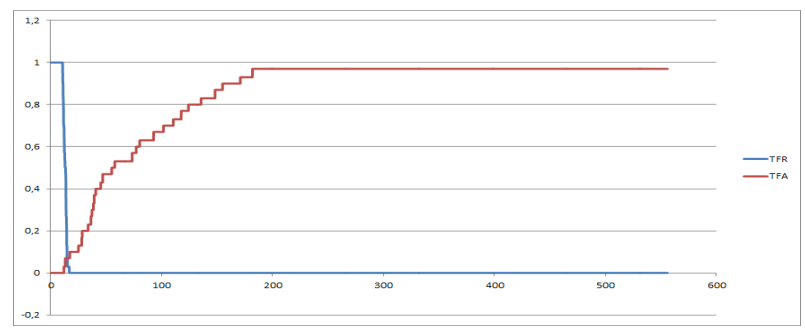

Figura 2: Taxa de Falsa Aceitação e Falsa Rejeição.

com objetivo de melhorar a qualidade da imagem de impressão digital de entrada (uniformizar o contraste, reduzir ruídos, remover imperfeições, realçar as características), extrair as minúcias, preparar o modelo de dados e reconhecer o datilograma. A metodologia apresentada neste trabalho constitui um sistema completo de identificação via imagens digitais, o que difere da maioria das metodologias descritas na literatura, que apresentam apenas algumas das etapas do processamento.

Por fim, o algoritmo proposto mostrou-se eficiente e robusto, no sentido de que possui um resultado superior à maioria daqueles apresentados na Tabela 2.

\section{Referências}

[1] J. C. Amengual, A. Juan, J. C. Prez, F. Prat, S. Sez and J. M. Vilar, "Real-time minutiae extraction in fingerprint images", in Proc. of the 6th Int. Conf. on Image Processing and its Applications, 1997, pp. 871-875.

[2] A. M. Bazen and S. H. Gerez, "Segmentation of Fingerprint Images", Workshop on Circuits, Systems and Signal Processing, 2001.

[3] D. Gabor, "Theory of Communication", Journal of Institute of Electrical Engineers, Vol. 93, pp. 429-457, 1946.

[4] V. Govindaraju and, T. Jea, "Minutiae-based partial fingerprint recognition", Pattern Recognition, Vol. 38, pp. 1672-1684, USA, 2005.

[5] C. J. HILDITCH, "Linear Skeletons from Square Cupboards", Machine Intelligence, 1969.

[6] L. Hong, Y. Wan and A Jain, "Fingerprint Image Enhancement: algorithm and performance evaluation", Transactions on Pattern Analysis and machine Intelligence, IEEE, 1998.

[7] D. Jomaa, "Segmentation of Low Quality Fingerprint Images", Multimedia Computing and Information Technology (MCIT), 2010.

[8] P. R. Kumar, "Watermarking Algorithm Using Sobel Edge Detection", Int. J. Advanced Networking and Applications, Volume: 02, Issue: 05, Pages: 861-867, 2011.

[9] D. Maltoni and D. Maio, "Fingerprint Verification Competition", 2006, Disponível em "http://bias.csr.unibo.it/fvc2006/results/O_res_db2_a.asp".

[10] M. Tico and P. Kuosmanen, "An Algorithm for Fingerprint Image Postprocessing", Proc. 34th Asilomar Conf. Signals, Systems and Computers, vol. 2, pp. 1735-1739, 2000. 